\title{
Rectal foreign bodies: retained orange
}

\author{
Francesk Mulita, George Theofanis, Levan Tchabashvili, Nikolas Drakos, Ioannis Maroulis
}

Department of Surgery, General University Hospital of Patras, Patras, Greece

Gastroenterology Rev 2021; 16 (4): 392-393

DOI: https://doi.org/10.5114/pg.2021.105409

\begin{abstract}
Address for correspondence: Dr. Francesk Mulita, Department of Surgery, General University Hospital of Patras, Greece, e-mail: oknarfmulita@hotmail.com
\end{abstract}

Cases with rectal foreign bodies are no longer rare; however, epidemiological data even nowadays are lacking [1]. The first report was published in 1919, although cases have been described since the $16^{\text {th }}$ century [2]. These patients usually present to the emergency department with complains of pelvic or lower abdominal pain [1]. Although there are a wide variety of types of foreign bodies and reasons for insertion, household objects such as bottles or glasses for autoeroticism is one of the most common reasons [2].

An otherwise healthy 53-year-old male presented to the emergency department with complaints of lower abdominal pain and discomfort from a large round-shaped orange he had inserted in his rectum approximately $12 \mathrm{~h}$ prior to presentation. His attempts to remove it at home failed prior to his arrival at the emergency department. On examination, the patient's temperature was 36.4 , heart rate was 85 beats per minute, blood pressure was 138/91 $\mathrm{mm} \mathrm{Hg}$, and respi-

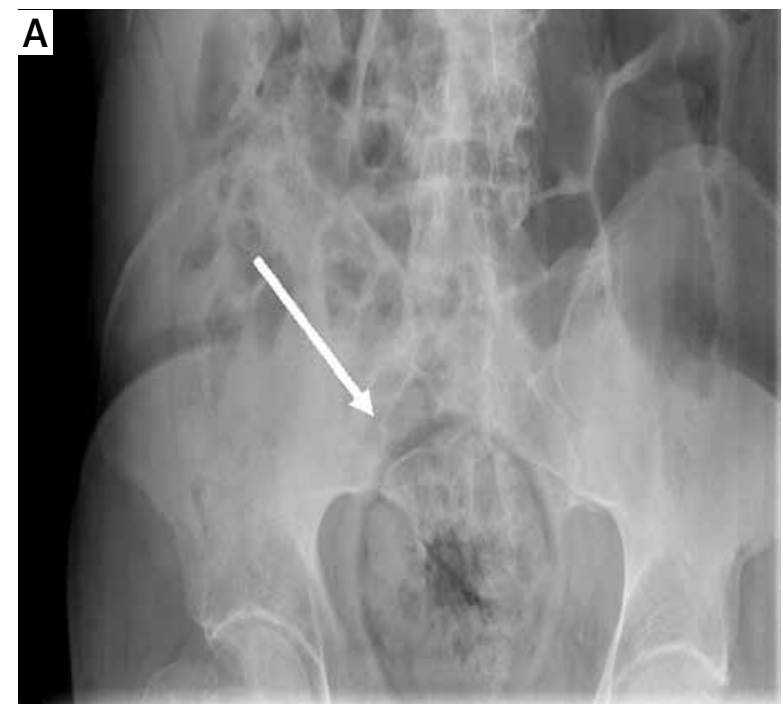

ratory rate was 17 breaths per minute. His abdomen was soft and non-distended, and mild tenderness was demonstrated in the left lower quadrant, without signs of peritonitis. The foreign body was palpable in the rectum and no blood was found on the examining glove. His routine blood tests including haemogram, C-reactive protein level, liver, and renal function test were normal. Abdominal radiography revealed a smooth, rounded, radio-dense foreign body within the midline of pelvis (Figures $1 \mathrm{~A}, \mathrm{~B}$ ), and there was no evidence of free air.

Because the patient was clinically stable and without evidence of perforation or peritonitis, attempts were made to manually remove the foreign body in the emergency department using anal dilators, forceps and proctoscopy. This was unsuccessful, and as a result of our manoeuvres, the foreign body was pushed higher into the rectum beyond the reach of the examining finger and instrument. The patient was taken to the operating room for examination after anaesthesia and

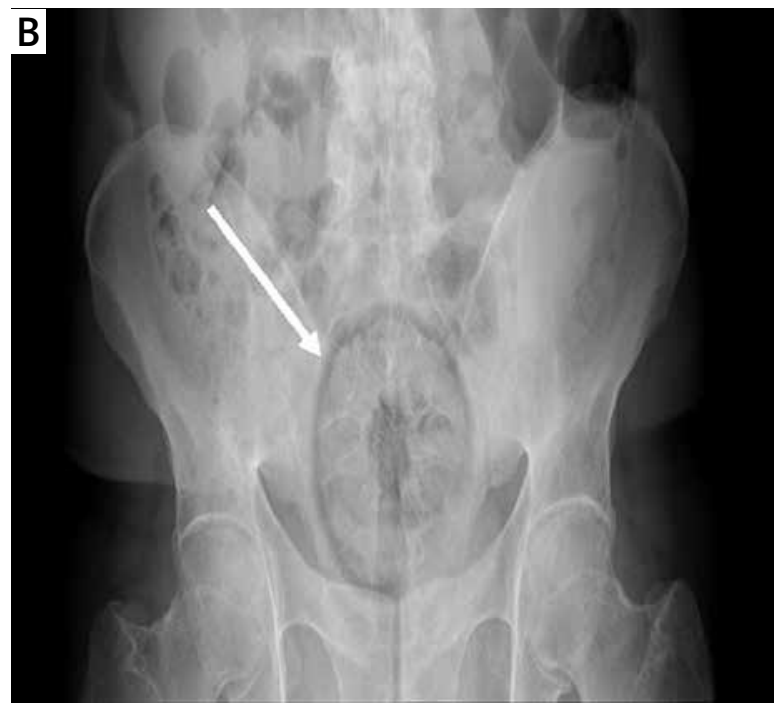

Figure 1. Abdominal radiograph. A - Supine position, B - erect position. Abdominal radiograph showing a smooth, rounded, radio-dense foreign body within the midline of the pelvis 


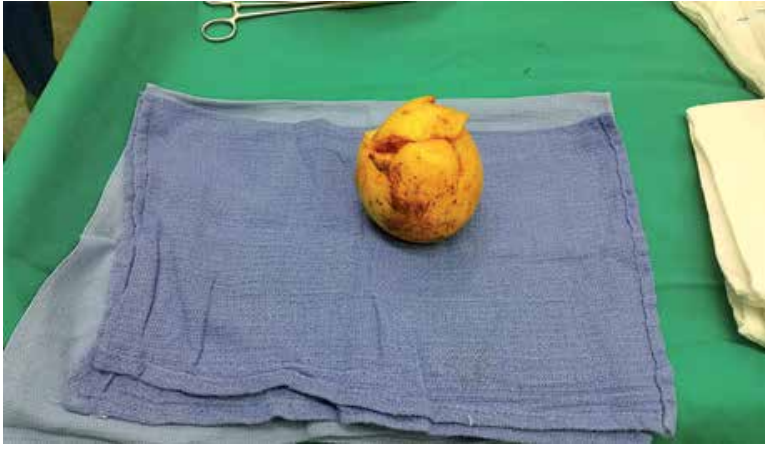

Figure 2 .This single trial succeeded, and the foreign body was identified as a 10 -cm-long orange

possibly surgical exploration. He was placed in the lithotomy position with reverse Trendelenburg angulation. The surgeon attempted to remove the object manually with lubrication and by using forceps, while suprapubic pressure was applied by an assistant to help move the foreign body. This single trial succeeded, and the foreign body was identified as a 10-cm-long orange (Figure 2). Post extraction, the patient did not have any complications and no abnormality was detected in sigmoidoscopy and abdominal X-ray. He had no abdominal pain and was discharged in a good condition.

Rectal body insertion has been described in the surgical literature since the $16^{\text {th }}$ century [3]. Patients with a retained rectal foreign body are often ashamed of their diagnosis, and it is very important that clinicians speak respectfully with these patients in order to build a trust-based relationship [4]. The good history of the patient and the physical exam including abdominal exam and digital rectal exam are the key points for the diagnosis of such a situation. An abdominal radiograph will confirm the diagnosis [5]. In a stable patient, the foreign body can be extracted in the emergency department transanally with a regional block and/or sedation [6]. Endoscopy can be helpful in cases where the foreign body is located high in the rectum or in the colon [3]. If these fail or the patient is haemodynamically unstable, then he/she should be transported to the operating room for further treatment $[3,7]$.

\section{Conflict of interest}

The authors declare no conflict of interest.

\section{References}

1. Ayantunde AA, Unluer Z. Increasing trend in retained rectal foreign bodies. World J Gastrointest Surg 2016; 8: 679-84.

2. Cologne KG, Ault GT. Rectal foreign bodies: what is the current standard? Clin Colon Rectal Surg 2012; 25: 214-8.
3. Kasotakis G, Roediger L, Mittal S. Rectal foreign bodies: a case report and review of the literature. Int J Surg Case Rep 2012; 3: 111-5.

4. Cawich SO, Thomas DA, Mohammed F, et al. A management algorithm for retained rectal foreign bodies. Am J Mens Health 2017; 11: 684-92.

5. Ayantunde AA. Approach to the diagnosis and management of retained rectal foreign bodies: clinical update. Tech Coloproctol 2013; 17: 13-20.

6. Bakheit IA, Elhasan GAB, Salih MA. A case of impacted foreign body in the rectum that was extracted using size 24 Foley catheter. Int J Surg Case Rep 2020; 66: 146-8.

7. Ologun GO, Stevenson Y, Cagir B, et al. Successful retrieval of a retained rectal foreign body in the emergency department. Cureus 2018; 10: e2025.

Received: 21.05 .2020

Accepted: 22.03 .2021 